\title{
Comparison of the Matching of Keywords of Abstracts of Articles Latin Selected Journals of Iranian Dental Indexed in Pubmed with Medical Subject Headings (MeSH)
}

\author{
Seyed Javad Ghazi-Mirsaeid ${ }^{1}$, Fatemeh Masoudi*2 \\ 1. Associate Professor of Medical Library and Information Science Department, School of Allied Medical \\ Sciences and Member of Health Information Management Researchs Center, Tehran University of \\ Medical Sciences, Tehran, Iran \\ 2. MSc, Medical Library and Information Science, School of Allied Medical Sciences, Tehran University \\ of Medical Sciences, Tehran and Librarianship, Iran Public Libraries Foundation, Qom, Iran \\ *(Corresponding Author) Email: f.masodi@qompl.ir
}

Abstract:- Background: This study evaluates the quality of keywords of abstracts of Latin Selected Journals of Iranian Dental indexed in PubMed with Medical Subject Headings (MeSH).

Materials and Methods: The study is comparative descriptive. The data gathering tool is a researcher-made checklist. The Statistical population of the study is two Latin Journals of Iranian Dental approved by the Ministry of Health that indexed in PubMed. The Access 2013 software was used to describe data.

Results: In total, 866 keywords of 208 articles were studied. On average, 4.2 keywords were in per article of selected Journals. The amount of the Exact match, Relatively match and Not match keywords in selected Journals is $39.5 \%, 35.8 \%$ and $24.8 \%$ respectively. More keywords of articles of both selected journals are the type of partial match with MeSH Headings or Entry Terms, and then keywords that are consistent with Entry Terms.

Conclusions: Despite the higher rate of consistent keywords of articles of the journal "J Dent (Tehran)" to the journal "Dent Res J (Isfahan)", the precision in the use of MeSH in both of the selected journal was not at a desirable level and the authors of the selected journals must have more attention in selecting their words index based on MeSH.

Key Words: Medical Subject Headings, Controlled Vocabulary, PubMed, Medical Abstracting and Indexing, Dentistry.

\section{INTRODUCTION}

All research articles are initiated by titles that followed by the abstract and a number of keywords. These three characteristics describe the content of an article [1]. The title provides snapshots of the content of the work in a concise manner. The abstract provides a summary of the content and keywords show core concepts and key areas of the paper. The four basic elements of a paper title, author, abstract and keywords in the design of each bibliographic databases is required to help electronic data recovery [2]. In fact, add keywords to the end of the abstract of articles are done with two major goals: 1 . To reveal the subject structure of article 2 . Provide an index Search [3]. That's why one of the important items when submitting a work for publication that authors are bound to it is typing keywords or descriptors associated with the work [4]. A proper select of keywords helps the paper to be in the direction of recovery, especially in an online search on the Internet [5]. Indexes are one of the most effective tools to identify, organize, retrieve and disseminate information [6] which have been established on the basis of allocated appropriate keywords to describe the document as a connection point the user to the desired document among a host of irrelevant and relevant issues. Therefore, the Keywords are the indexes in a scientific text. Keywords are used for the classification of articles in some databases and if an author has selected the better and more accurate keywords the probability that his article is read by those who work on their paper work will be more [7]. Paper that has been selected by poor keyword or with a subject has not been described is as a missing star in the sky that any scientist or poet will not refer to it [8]. Then indexing of medical records is important to retrieve medical information [9]. Indexing in biomedical research is means the using of a thesaurus National Library of Medicine of America: Medical Subject Headings (MeSH), which as a standard has been accepted by libraries and medical journals around the world, and provides quick and easy access to detailed information using of keywords assigned to any document [10].

Certainly one of the sciences that because deal with life and death issue of people have a special place, and due to that reason needs to turn more information than any other science is medical science and related sciences to it.On the other hand, among the related disciplines to medical sciences, dentistry due to awareness of status of mouth and tooth health that are critical organs in human life has a great importance because of the help 
of the science many diseases are recognized at the early stages and their progression is prevented [11]. Hence the importance of publishing scientific paper and making available the results of research and experiences of researchers in this area to improve the quality of health services is obvious. Given that as yet a research within compare keywords with MeSH among the English online documentation of Iranian journals indexed in PubMed has not been done, This study attempts to monitor and identify the quality level of the inserted keywords at the end of the abstract of articles of selected Iranian Latin Dental Journals indexed in PubMed leads to the awareness that the scientific literature of dentistry published at the base is on the line to recovery and citation, and therefore in a cycle of information or not.

\section{MATERIALS AND METHODS}

The study is cross-sectional. The population of this study are two journals (bimonthly) "Dent Res $\mathbf{J}$ (Isfahan)" and "J Dent (Tehran)" Iranian Latina journal approved by the Medical Journals Commission (Ministry of Health and Medical Education) that from the beginning of publication until the end of 2014 were indexed in PubMed. The data gathering tool is a researcher-made checklist. Collecting and extracting keywords with refer to any number of the two journal and its articles in PubMed database was performed. The inserted keywords at the end of the abstract of all types of existing articles in the two journal with refer to link of the two journal in PubMed database were extracted and were entered to checklist designed researcher in Access 2016 software. In order to adapt keywords with MeSH, the online format MeSH (MeSH browser 2014) was used. All of keywords are classified to three large categories; Exact match (Complete coincidence between keyword and MeSH Headings), Relatively match (Difference in separation with MeSH Heading, Difference in Plural or singular with MeSH Heading, Difference in the order of words with MeSH Heading, Entry Terms, Difference in separation with Entry Terms, Difference in Plural or singular with Entry Terms, Difference in the order of words with the Entry Terms, Partial Match with MeSH Heading or Entry Terms) and Not match (Lack of Keywords in MeSH).

\section{RESULTS}

A total of 113 articles with 474 keywords of "Dent Res J (Isfahan)" and 95 articles with 392 keywords of "J Dent (Tehran)", was published in 2014. The average number of keywords in each article for both journals is about 4.2 keywords. The greatest number of keywords used in both the journal is 8 and the lowest number of keywords used in both the journal is 3 . In both of these journals three types of paper, including review articles, research papers and case reports were observed. The findings of this study showed that, after research articles that form large volume of articles published of the two journals indexed in PubMed, case reports are the most articles and review articles are the lowest. 75.5\% keywords of articles of the journal "Dent Res J (Isfahan)" and $80.4 \%$ keywords of articles of the journal "J Dent (Tehran)" are in research articles and about $20 \%$ to $25 \%$ keywords are in review articles and case reports.

The Review of articles of the two journal based on the pattern of the number of inserted keywords at the end of their abstract showed that of a total of 208 article under investigation, articles with 3 and 4 keywords with $66.8 \%$ are the most and articles with 7 and 8 keywords with $2.9 \%$ are the lowest. More articles of "Dent Res J (Isfahan)" with the insertion of 4 keywords and more articles of "J Dent (Tehran)" by inserting 3 keywords at the end of the abstract of the article were written. The minimum and maximum number of keywords used in articles of the two journals is 3 and 8 keywords respectively (Table 1).

Table 1. Frequency of articles based on the pattern of the number of keywords in each article

\begin{tabular}{|c|c|c|c|c|c|c|}
\hline $\begin{array}{c}\text { Number of keywords } \\
\text { in article }\end{array}$ & $\mathbf{3}$ & $\mathbf{4}$ & $\mathbf{5}$ & $\mathbf{6}$ & $\mathbf{7}$ & $\mathbf{8}$ \\
\hline Journal title & $29.2 \%$ & $37.2 \%$ & $23.9 \%$ & $6.2 \%$ & $1.8 \%$ & $1.8 \%$ \\
\hline Dent Res J (Isfahan) & $35.8 \%$ & $31.6 \%$ & $21.1 \%$ & $9.5 \%$ & $0.0 \%$ & $2.1 \%$ \\
\hline J Dent (Tehran) & $32.2 \%$ & $34.6 \%$ & $22.6 \%$ & $7.7 \%$ & $1 \%$ & $1.9 \%$ \\
\hline Total & & &
\end{tabular}

Evaluation of the using of MeSH by authors of selected journal articles in the field of dentistry by comparing keywords inserted at the end of the abstract of their articles with MeSH showed that of a total of 866 keywords inserted at the end of the abstract of 208 article of the two journal, 39.5\% are Exact match with the $\mathrm{MeSH}, 35.7 \%$ have Relatively match with the MeSH and $24.8 \%$ have Not match with MeSH (Table 2). 
Table 2. Frequency of the amount of the Exact Match, Relatively Match and Not Match keywords in selected journals

\begin{tabular}{|c|c|c|c|}
\hline $\begin{array}{c}\text { Categories of Match } \\
\text { Journal title }\end{array}$ & Exact Match & Relatively Match & Not match \\
\hline Dent Res J (Isfahan) & $33.8 \%$ & $33.3 \%$ & $32.9 \%$ \\
\hline J Dent (Tehran) & $46.4 \%$ & $38.5 \%$ & $15.1 \%$ \\
\hline Total & $39.5 \%$ & $35.7 \%$ & $24.8 \%$ \\
\hline
\end{tabular}

Keywords that were Relatively match with MeSH based on the conflict that have with MeSH Headings or Entry Terms were classified into 8 groups. Results showed that the first rank among the types of Relatively Match is owned by keywords that part of them with MeSH Headings, or its synonyms are consistent. This amount with the $1.5 \%$ differences in keywords of articles of the journal "J Dent (Tehran)", more than keywords of articles of

the journal "Dent Res J (Isfahan)" can be seen. These keywords form two-fifths of the Relatively Match keywords of articles of selected journals. After that keywords with synonyms (Entry Terms) existing in MeSH are match not with the headings listed in the MeSH have highest frequency of Relatively Match keywords, this keywords form about two-thirds of the Relatively Match keywords of articles of selected journals, this type of Relatively Match with 7\% difference in Relatively Match keywords of article of the journal "J Dent (Tehran)", more than the Relatively Match keywords of "Dent Res J (Isfahan)" can be seen (Table 3).

Table 3. Frequency of keywords in terms of Relatively Match in selected journals

\begin{tabular}{|c|c|c|c|c|c|c|c|c|}
\hline $\begin{array}{c}\text { Categories of Relatively } \\
\text { Match }\end{array}$ & 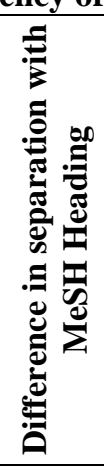 & 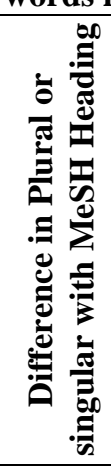 & 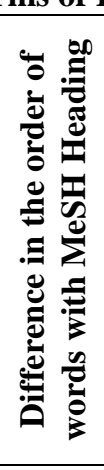 & 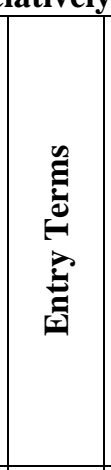 & 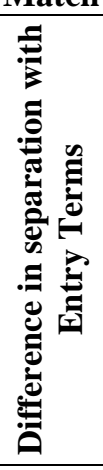 & 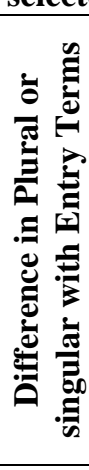 & 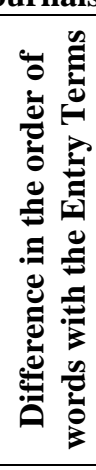 & 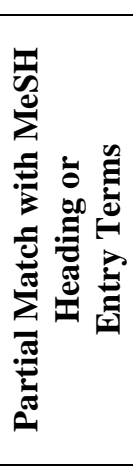 \\
\hline Dent Res J (Isfahan) & $2.5 \%$ & $9.5 \%$ & $5.1 \%$ & $26.6 \%$ & $0.6 \%$ & $9.5 \%$ & $1.3 \%$ & $44.9 \%$ \\
\hline J Dent (Tehran) & $1.3 \%$ & $15.2 \%$ & $0.0 \%$ & $33.8 \%$ & $0.0 \%$ & $2.6 \%$ & $0.7 \%$ & $46.4 \%$ \\
\hline Total & $1.9 \%$ & $12.3 \%$ & $2.6 \%$ & $30.1 \%$ & $0.3 \%$ & $6.1 \%$ & $1 \%$ & $45.6 \%$ \\
\hline
\end{tabular}

\section{DISCUSSION}

In the directory of the journal "Dent Res J (Isfahan)", the number of permitted keywords to describe the document is 3 to 5 and in the directory of the Journal "J Dent (Tehran)", the number of permitted keywords to describe the document is 3 to 7 . While the results of this study showed that, 3 to 8 keyword has been used to describe the articles of the selected journal. 9.8\% of articles of the journal "Dent Res J (Isfahan)" and 2.1\% of articles of the journal "J Dent (Tehran)" have been described with the number more than the number of keywords permitted in the directory for authors.

Although the inconsistencies found in describing Keywords of articles of selected journals is less than $10 \%$, this inconsistency in the articles of the journal of "Dent Res J (Isfahan)", more than articles of the journal "J Dent (Tehran)", can be seen. If this is expected that authors, consider a paragraph related to allowed number of keywords to describe the article in the directory for authors and the result is a more coordination between the applied keywords by authors in articles with the number stated in the directory for authors.

In relation with keywords match with MeSH also results showed almost the same level of the Exact match, Relatively Match and Not Match keywords with MeSH in articles of the journal "Dent Res J (Isfahan)". While the amount of the Exact match, Relatively keywords of articles of the journal "J Dent (Tehran)" is more than its Not match keywords and in each three keyword of articles of the journal has exist an Exact match keyword, a Relatively match keyword, and a Not Match keyword with the MeSH. Also the amount of Not Match keywords of articles of the journal "J Dent (Tehran)" is less than the half of Not Match keywords of articles of the journal "Dent Res J (Isfahan)".

And this show that authors of articles of the journal "J Dent (Tehran)" have consider the other paragraph in relation with the use of MeSH to select keyword of article more successful than his counterpart journal. The results showed that the total amount of the Exact match, Relatively Match and Not Match keywords 
of articles of selected journals are $39.5 \%, 35.7 \%$ and $24.8 \%$ respectively that generally indicates the degree of Exact match is not at a high level. The findings of the studies of Botelho \& Teixeira [12], Teixeira and colleagues [13] found inconsistent results with the study. They argued that the use of keywords out of MeSH is not consistent with policy of journal.

The findings of Kim et al [14], Kim et al [15], Roh [16], Naghneh Esfahani et al [17], Aram [18], Kabiri Zadeh and colleagues [19], Bahadori and Bani Eghbal [20], Islami and Bigdeli [21] reached to the results that they are similar to this study and many reasons, including: not recognition of the MeSH by writers, lack of use, lack of attention to MeSH, the lack of standardization of indexes based on MeSH and etc, are the reasons for a low level of Exact match keywords with MeSH. Based on this study results, searchers for articles of the journals in PubMed database in addition to the use of MeSH must use the keywords of natural language when searching to articles be retrieved.

The presence of more than one-third of keywords with varying degrees of Relatively Match with MeSH shows in addition to the use of MeSH to find articles, search must be done with keywords with a variety of Relatively Match with the MeSH in order to ensure the desired result. Among the keywords with Relatively Match with MeSH, Exact match keywords with MeSH or Entry Terms with the difference in plural and singular, Exact match keywords with Entry Terms and keywords with having more or less terms than the MeSH or Entry Terms form $12.3 \%, 30.1 \%$ and $45.6 \%$ of the total of Relatively Match Keywords with MeSH that basis using of such keywords when searching for articles increases the chances of recovery and achieve Articles in the same amount.

The result of study of Kim et al [15] also showed Exact match keywords with Entry Terms, different keywords in plural or singular than MeSH or Entry Terms and keywords with the difference in separation with MeSH or Entry Terms form, 1.6\%, $44.6 \%$ and $38.5 \%$ of the total of Relatively Match keywords respectively. Kang et al [22] also represented in their study the amount of the Exact match keywords with Entry Terms is $13.9 \%$, and the amount of different keywords in plural or singular than MeSH or Entry Terms and the amount of keywords with less or more terms than MeSH are 1\% and 19.4\% respectively. Roh [16], also showed that the amount of Exact match keywords with Entry Terms, although is very little but exist in his study, the keywords with more or less terms than MeSH have formed the maximum amount of Exact match keywords with MeSH.

In fact, this study shows that the accuracy of authors in correct and error-free use of MeSH causes that $\mathrm{MeSH}$ continue to be the most appropriate tool used to retrieve information. Gross and colleagues [23] represented in the absence of controlled vocabulary are many works that will not retrieve. He proposed regarding to the effectiveness of the use of controlled language, it is necessary to help new users in order to take advantage of controlled words with creating tools.

\section{CONCLUSION}

The amount of Exact match keywords with MeSH in both the journal is less than 50\% that shows the amount of Exact match keywords is not at desirable level and in indexing these works has not been followed the same policy. The reasons, including a lack of fluency in the process of selecting keyword from MeSH, failure to recognize the differences between accepted headings and its synonyms and inaccuracy of authors in correct writing keyword after selection a keyword of MeSH leads to conflict the keywords with MeSH. This shows necessary training and the practices of use of documentary tools such as Medical Subject Headings and Thesaurus have not a place in curricula of the dental community users and it seems that a lot of authors only when submitting the paper to the journal notice that is required to use MeSH, obviously at the time of submission, the author will not be looking to learn how to select keywords from MeSH and since the education of using of the MeSH have not been put in the directory for authors of articles of selected journal, this leads to low quality keywords of articles.

\section{ACKNOWLEDGEMENT}

This manuscript is based on a thesis (Thesis number: 280/3/K/67) submitted to the graduate faculty, School of Allied Medical Sciences, Tehran University of Medical Sciences, Tehran, Iran, in partial fulfillment of the requierments for Master of Science (MSc) Degree.

\section{REFERENCES}

[1] Hartley J, Kostoff RN. How useful are "Key Words" in scientific journals? J. Inf. Sci. 2003 Oct; 29 (5): 433-8.

[2] Dutta B, Majumder K, Sen B. Classification of keywords extracted from research articles published in science journals. Ann. Libr. Inf. Stud. 2008 Oct; 55 (4): 317-33.

[3] Hamidiyeh M. Keyword. Mahfel. 2012; 1 (1): 42-44.

[4] Brandau R, Monteiro R, Braile DM. Importance of the correct use of descriptors in scientific articles. Rev. Bras. Cir. Cardiovasc. 2005 Mar; 20 (1): VII - IX. 
[5] Ng K, Peh W. Effective Medical Writing. Singap. Med J. 2008; 49 (12): 967-9.

[6] Noruzi A, Velayati K. Subject indexing concept indexing. Tehran: Chapar Publication; 2010. 281 p.

[7] Habibi E, Mirhosseini Z. writing articles with criteria ISI (Institute for Scientific Information). Knowl. Sci. Libr. Inf. Sci. Technol. 2008; 1 (2): 51-62.

[8] Timi JRR. A importância do uso dos descritores nas publicações médicas. J Vasc Bras. 2005 Jun; 4 (2):114-5.

[9] Lee JS, Oh YS, Han SH, Lee YS. A Korean MeSH keyword suggestion system for medical paper indexing. J. Korean Soc. Med. Inform. 2005 Dec; 11 (4): 337-43.

[10] Brkić S, Vučenović M, Đokić Z. Title, abstract, key words and references in biomedical articles. Arch. Oncol. 2003 Sep; 11 (3): 207-9.

[11] Dentistry in Iranian universities. Book Mon Sci Technol. 2010 Sep-Oct; 4 (6): 14-7.

[12] Teixeira RKC, Botelho NM. Avaliação dos descritores utilizados em artigos publicados em dois periódicos nacionais sobre cirurgia. Rev Para Med. 2011 Sep; 25(2/3).

[13] Teixeira RKC, Gonçalves TB, Yamaki VN, Botelho NM, Brito MVH. Evaluation of the key words used in articles of the Acta Cirurgica Brasileira from 1997 to 2012. Acta Cir. Bras.2012 May; 27 (5): 350-4.

[14] Kim E-J, Ma D-S, Jin B-H. An analysis of the articles published in the Journal of Korean Academy of Oral Health, from 1995 to 2012. J. Korean Acad. Oral Health 2013 Mar; 37 (1): 53-8.

[15] Kim Y-Y, Park H-J, Lee S-W, Yoo J-H. Comparison of Keywords of the Journal of Sasang Constitutional Medicine with MeSH Terms. J. Sasang Const. Med. 2013; 25 (1): 34-42.

[16] Roh J-S. The Comparison of Keyword of Articles in Journal of the Korean Society of Physical Medicine with MeSH. J. Korean Soc. Phys. Med. 2012 Aug; 7 (3): 367-77.

[17] Naghneh Esfahani M, Cheshmeh Sohrabi M, Banieghbal N. A Comparative Study of the Persian and English Keywords of Theses from the Isfahan University of Medical Sciences, Iran, and the Thesauruses and Persian Medical Subject Headings. Health Inf. Manag. 2013; 9 (6): 813.

[18] Aram F. Comparative Study Keywords Theses Zahedan University of Medical Sciences with Medical Subject Headings (MeSH) in the period 1380-1391 [Master's thesis]. [Tehran]: Islamic Azad University, North Tehran Branch; 2014.

[19] Kabirzadeh A, Siamian H, Abadi EB, Saravi BM. Survey of keyword adjustment of published articles medical subject headings in journal of mazandaran university of medical sciences (2009-2010). Acta Inf. Med 2013 Apr; 21 (2): 98-102.

[20] Bahadori L, Banieghbal N. Adaptation of Descriptors and Keywords of Medical Thesis of Tehran University of Medical Science to Persian Medical Thesaurus and Medical Subject Headings. J. Epistemol. Libr. Inf. Sci. Inf. Technol. 2014; 7 (24): 53-68.

[21] Eslami A, Bigdeli Z. The Investigation of the Correlation Rate of the Keywords of Persian Articles in Medical Journals with Medical Persian Thesaurus During the Years 2008 and 2009. Indian J. Fundam. Appl. Life Sci. 2014 Mar; 4 (3): 1132-41.

[22] Kang K-B, Kim J-H, Kim YB, Kim J-K, Shin S-M. Use of Medical Subject Headings (MeSH) in the Journal of the Korean Orthopaedic Association. J. Korean Orthop. Assoc. 2014 Aug; 49 (4): 302-6.

[23] Gross T, Taylor AG, Joudrey DN. Still a Lot to Lose: The Role of Controlled Vocabulary in Keyword Searching. Cat. Classif. Q. 2015 Jan; 53 (1): 1-39. 